\title{
One-loop correction to the energy of a wavy line string in $A d S_{5}$
}

\author{
E. I. Buchbinder \\ School of Physics M013, The University of Western Australia, \\ 35 Stirling Highway, Crawley W.A. 6009, Australia \\ A. A. Tseytlin* \\ The Blackett Laboratory, Imperial College, London SW7 2AZ, U.K.
}

\begin{abstract}
We consider a computation of one-loop $A d S_{5} \times S^{5}$ superstring correction to the energy radiated by the end-point of a string which moves along a wavy line at the boundary of $A d S_{5}$ with a small transverse acceleration (the corresponding classical solution was described by Mikhailov in hep-th/0305196). We also compute the one-loop effective action for an arbitrary small transverse string fluctuation background. It is related by an analytic continuation to the Euclidean effective action describing one-loop correction to the expectation value of a wavy Wilson line. We show that both the one-loop contribution to the energy and to the Wilson line are controlled by the subleading term in the strongcoupling expansion of the function $B(\lambda)$ as suggested by Correa, Henn, Maldacena and Sever in arXiv:1202.4455.
\end{abstract}

${ }^{*}$ Also at Lebedev Institute, Moscow 


\section{Introduction}

In [1] it was suggested that there is universality in a class of near-BPS problems: expectation values of small-cusp Euclidean Wilson loop, Euclidean wavy Wilson line, energy radiated by a moving particle in Minkowski space. They all are controlled by the same function $B(\lambda)$

$$
B(\lambda)=\frac{\sqrt{\lambda}}{4 \pi^{2}} \frac{I_{2}(\sqrt{\lambda})}{I_{1}(\sqrt{\lambda})}=\frac{\sqrt{\lambda}}{4 \pi^{2}}-\frac{3}{8 \pi^{2}}+\frac{3}{32 \pi^{2} \sqrt{\lambda}}+\ldots
$$

In the generalized cusp case the corresponding one-loop strong-coupling corrections were already discussed in [2]. In this paper we attempt to further check this prediction at strong coupling in the case of radiation and wavy line, reproducing the first subleading coefficient in (1.1) by a direct one-loop superstring computation.

We shall start in section 2 with a review of a classical string solution in Minkowski-signature $A d S_{5}$ ending on a nearly-straight time-like line at the boundary. It is described in the static gauge by the Mikhailov's solution [3] (see also [4]). We compute the classical string action and the energy on this solution. In the remaining sections we shall compute the one-loop corrections to these classical expressions.

In section 3 we shall first consider the contribution from the bosonic string fluctuations using static gauge. Similar computations (see $[5,6,7]$ ) involve several subtle points that we will not directly address here (assuming cancelation of UV divergences and conformal anomaly should happen in the full theory as it does in the conformal gauge). In particular, we will thus ignore the one-loop path integral over the $S^{5}$ string modes as it should contribute only to the divergences and the conformal anomaly.

In section 4 we will compute the one-loop fermionic contributions. We avoid detailed study of cancellation of UV divergences by using a heuristic (but natural) argument of how to extract the relevant finite contribution. Summing up the bosonic and the fermionic contributions we find that the full one-loop correction to the radiated energy is indeed proportional to the nextto-leading coefficient in the expansion in (1.1).

Finally, in section 5 we will consider the Euclidean wavy Wilson line solution $[8,9,10]$. The one-loop correction to the expectation value of the wavy Wilson line is given by the Euclidean one-loop effective action which can be obtained by an analytic continuation from the corresponding Minkowski-signature expression computed in Sections 3 and 4. As a result, we find that this one-loop correction is also governed by the next-to-leading coefficient in (1.1). 


\section{Classical solution for a string ending on a wavy line}

We shall parametrize the worldsheet by $(\tau, \sigma)$ and $A d S_{5}$ by the Poincare coordinates

$$
\begin{aligned}
& \left(X^{\mu}, Z\right)=\left(X^{0}, X^{i}, Z\right), \quad \mu=0, \ldots, 3, \quad i=1,2,3, \\
& d s^{2}=\frac{-\left(d X^{0}\right)^{2}+\left(d X^{i}\right)^{2}+d Z^{2}}{Z^{2}} .
\end{aligned}
$$

Except for the last section, in this paper both the worldsheet and $A d S_{5}$ are assumed to have Minkowski signature with $\tau$ and $X^{0}$ being timelike. We will consider the static gauge

$$
X^{0}=\tau, \quad Z=\sigma .
$$

The Nambu-Goto action is then given by

$$
S_{c l}=-\frac{\sqrt{\lambda}}{2 \pi} \int d \tau d \sigma \sqrt{-g},
$$

where the induced metric is

$$
g_{\alpha \beta}=\frac{1}{Z^{2}} \eta_{\mu \nu} \partial_{\alpha} X^{\mu} \partial_{\beta} X^{\nu}=g_{0 \alpha \beta}+\frac{1}{\sigma^{2}} \partial_{\alpha} X^{i} \partial_{\beta} X^{i}, \quad \alpha, \beta=1,2
$$

with $g_{0 \alpha \beta}$ being the $A d S_{2}$ metric $g_{0 \alpha \beta}=\frac{1}{\sigma^{2}} \operatorname{diag}(-1,1)$,

The simplest solution is when a string end moves along a time-like straight line at the boundary $X^{i}(\tau, \sigma=0)=0$. We shall consider more general solution where a string end-point moves along small deviation of that straight line. To leading order in $X^{i}$ the string action (2.3) is given by

$$
S_{c l}=\frac{\sqrt{\lambda}}{4 \pi} \int d \tau d \sigma \frac{1}{\sigma^{2}}\left[\left(\partial_{\tau} X^{i}\right)^{2}-\left(\partial_{\sigma} X^{i}\right)^{2}\right]
$$

and the equation of motion is

$$
-\partial_{\tau}^{2} X^{i}+\partial_{\sigma}^{2} X^{i}-\frac{2}{\sigma} \partial_{\sigma} X^{i}=0
$$

Its solution with boundary conditions

$$
X^{i}(\tau, \sigma=0) \equiv \mathrm{x}^{i}(\tau)=\mathrm{x}_{+}^{i}(\tau)+\mathrm{x}_{-}^{i}(\tau)
$$

is given by [3]

$$
X^{i}=x_{+}^{i}(\tau+\sigma)-\sigma \dot{x}_{+}^{i}(\tau+\sigma)+x_{-}^{i}(\tau-\sigma)+\sigma \dot{x}_{-}^{i}(\tau-\sigma)
$$

where $x_{+}^{i}, x_{-}^{i}$ are arbitrary functions and by dot we denote the derivative over $\tau$. The solution is uniquely defined by the boundary curve $(2.7)$ the third normal derivative $\left.\left(\partial_{\sigma}^{3} X^{i}\right)\right|_{\sigma=0}[3]$. In this paper, for simplicity, we will set

$$
\mathrm{x}_{+}^{i}(\tau)=0, \quad \mathrm{x}_{-}^{i}(\tau)=\mathrm{x}^{i}(\tau) .
$$


We shall reserve the notation $x_{i}$ for $x_{-}^{i}(\tau-\sigma)$ using $\mathrm{x}_{\mathrm{i}}$ for the function $\mathrm{x}_{-}^{i}$ of $\tau$ only.

Let us evaluate the action and the energy on this solution in terms of the boundary data $\mathrm{x}^{i}(\tau)[3,4]$. Using equations of motion and ignoring the total $\tau$-derivative the classical action can be written as

$$
S_{c l}=\left.\frac{\sqrt{\lambda}}{4 \pi} \int \frac{d \tau}{\sigma^{2}}\left(X^{i} \partial_{\sigma} X^{i}\right)\right|_{\sigma=\epsilon} ^{\infty}=-\left.\frac{\sqrt{\lambda}}{4 \pi} \int \frac{d \tau}{\sigma^{2}} X^{i} \partial_{\sigma} X^{i}\right|_{\sigma=\epsilon},
$$

where we introduced the cut-off near the boundary and assumed that $X^{i}$ vanishes at infinity. Using the solution (2.8),(2.9) we obtain

$$
\frac{1}{\sigma^{2}} X^{i} \partial_{\sigma} X^{i}=-\frac{1}{\sigma} x^{i}(\tau-\sigma) \ddot{x}^{i}(\tau-\sigma)-\dot{x}^{i}(\tau-\sigma) \ddot{x}^{i}(\tau-\sigma) .
$$

Expanding $x^{i}(\tau-\sigma)$ near $\sigma=\epsilon \rightarrow 0$ we get

$$
\frac{1}{\sigma^{2}} X^{i} \partial_{\sigma} X^{i}=-\frac{1}{\epsilon} \mathrm{x}^{i}(\tau) \ddot{\mathrm{x}}^{i}(\tau)+\mathrm{x}^{i}(\tau) \dddot{\mathrm{x}}^{i}(\tau) .
$$

Substituting in (2.10) and integrating by parts gives

$$
S_{c l}=-\frac{\sqrt{\lambda}}{4 \pi} \frac{1}{\epsilon} \int d \tau\left(v^{i}\right)^{2}+\frac{\sqrt{\lambda}}{4 \pi} \int d \tau v^{i} a^{i}
$$

where

$$
v^{i}=\dot{\mathrm{x}}^{i}(\tau), \quad a^{i}=\ddot{\mathrm{x}}^{i}(\tau)
$$

are the velocity and the acceleration of the string's end-point. Hence, the finite part of the action is given by

$$
S_{c l, f i n}=\frac{\sqrt{\lambda}}{4 \pi} \int d \tau v^{i} a^{i},
$$

Now let us evaluate the classical energy. We define the target space energy as

$$
E_{c l}=-\int d \sigma \frac{\partial L}{\partial \partial_{\tau} X^{0}}
$$

where $L$ is the string Lagrangian in (2.3). Eq. (2.16) may be interpreted as the energy radiated by the end-point particle moving with acceleration. To compute (2.16) in static gauge (2.2) we have to restore the dependence on $X^{0}$ in the induced metric (2.4) and set $X^{0}=\tau$ after we take the derivative. Alternatively, since in the static gauge we have $X^{0}=\tau$, the energy (2.16) coincides with the two-dimensional Hamiltonian i.e.

$$
E_{c l}(\tau)=\frac{\sqrt{\lambda}}{4 \pi} \int \frac{d \sigma}{\sigma^{2}}\left[\left(\partial_{\tau} X^{i}\right)^{2}+\left(\partial_{\sigma} X^{i}\right)^{2}\right] .
$$

To find $E_{c l}$ in terms of the boundary data we first differentiate (2.17) with respect to $\tau$,

$$
\partial_{\tau} E_{c l}(\tau)=\frac{\sqrt{\lambda}}{2 \pi} \int \frac{d \sigma}{\sigma^{2}}\left[\partial_{\tau} X^{i} \partial_{\tau}^{2} X^{i}+\partial_{\sigma} X^{i} \partial_{\tau} \partial_{\sigma} X^{i}\right]
$$


then integrate by parts and then use equations of motion (2.6). As a result,

$$
\partial_{\tau} E_{c l}(\tau)=-\left.\frac{\sqrt{\lambda}}{2 \pi} \frac{1}{\sigma^{2}} \partial_{\tau} X^{i} \partial_{\sigma} X^{i}\right|_{\sigma=\epsilon} .
$$

From the solution $(2.8)$ we find that

$$
-\frac{1}{\sigma^{2}} \partial_{\tau} X^{i} \partial_{\sigma} X^{i}=\frac{1}{\sigma} \dot{x}^{i} \ddot{x}^{i}+\left(\ddot{x}^{i}\right)^{2}
$$

Expanding near the boundary $\sigma=\epsilon \rightarrow 0$ we obtain

$$
-\left.\frac{1}{\sigma^{2}} \partial_{\tau} X^{i} \partial_{\sigma} X^{i}\right|_{\sigma=\epsilon}=\frac{1}{\epsilon} \dot{\mathrm{x}}^{i} \ddot{\mathrm{x}}^{i}-\dot{\mathrm{x}}^{i} \dddot{\mathrm{x}}^{i}=\frac{1}{\epsilon} v^{i} a^{i}-\partial_{\tau}\left(v^{i} a^{i}\right)+\left(a^{i}\right)^{2} .
$$

Ignoring the divergence and the total derivative we find that the energy radiated over some period $\Sigma$ is given by

$$
E_{c l}=\frac{\sqrt{\lambda}}{2 \pi} \int_{0}^{\Sigma} d \tau\left(a^{i}\right)^{2}=2 \pi B_{0}(\lambda) \int_{0}^{\Sigma} d \tau\left(a^{i}\right)^{2}
$$

where to leading order in large tension expansion the coefficient $B(\lambda)$ is thus given by

$$
B_{0}(\lambda)=\frac{\sqrt{\lambda}}{4 \pi^{2}}
$$

The above classical consideration can be extended to the general case of non-linear dependence on $X^{i}[3,11,12]$ but it will not be discussed here.

\section{The one-loop correction in the bosonic sector}

Let us now consider the one-loop corrections to (2.13), (2.22). We will compute the oneloop effective action for an arbitrary transverse background $X^{i}(\tau, \sigma)$ that solves the linearized equations of motion (2.6). Let us reserve the notation $X^{\mu}$ for the classical background and denote the full $A d S_{5}$ string fields as $\left(Y^{0}, Y^{i}, Y^{4}\right)$, i.e.

$$
d s^{2}=\frac{-\left(d Y^{0}\right)^{2}+\left(d Y^{i}\right)^{2}+\left(d Y^{4}\right)^{2}}{\left(Y^{4}\right)^{2}},
$$

where $Y^{4}$ will be the radial direction. The $S^{5}$ string coordinates will be denoted as $\chi^{a}, a=$ $1, \ldots, 5$. We will impose the quantum static gauge ${ }^{1}$

$$
Y^{0}=X^{0}=\tau, \quad Y^{4}=Z=\sigma,
$$

\footnotetext{
${ }^{1}$ Like in a light-cone gauge, there is no non-trivial ghost determinant in a static gauge: this gauge is fixed directly in terms of string coordinates, but their variation under reparametrizations involve the gauge parameter only algebraically $\left(\delta X=\xi^{a} \partial_{a} X\right)$. The resulting ghost determinant is ultralocal, i.e. contributes only $\delta(0)$ terms. The same remark will apply to the $\kappa$-symmetry gauge we will use.
} 
i.e. the fields $Y^{0}, Y^{4}$ will not fluctuate. We will also split $Y^{i}$ as

$$
Y^{i}=X^{i}+\frac{y^{i}}{\lambda^{1 / 4}}
$$

where $y^{i}$ are the quantum fluctuations. Since the classical solution is non-zero only in $A d S_{5}$, the $S^{5}$ fields $\chi^{a}$ have only fluctuating part (which we also rescale by $\lambda^{-1 / 4}$ ). To compute the effective action to one-loop order we need to expand the Nambu-Goto action

$$
S_{b}=-\frac{\sqrt{\lambda}}{2 \pi} \int d \tau d \sigma \sqrt{-G}, \quad G_{\alpha \beta}=g_{\alpha \beta}(Y)+\frac{1}{\sqrt{\lambda}} \partial_{\alpha} \chi^{a} \partial_{\beta} \chi^{a},
$$

to quadratic order in the quantum fields $y^{i}, \chi^{a}$ (here $g_{\alpha \beta}$ is the induced metric depending on the $A d S_{5}$ fields $\left.Y^{m}\right)$.

Expanding (3.4) in powers of $y_{i}$ and $\chi_{a}$ we will get the classical action, then linear terms which vanish since the background satisfies the equations of motion, and, finally, the quadratic terms on which we will concentrate.

\subsection{The contribution from $S^{5}$}

The quadratic term in $\chi_{a}$ is

$$
S_{S^{5}}=-\frac{1}{4 \pi} \int d \tau d \sigma \sqrt{-g(X)} g^{\alpha \beta}(X) \partial_{\alpha} \chi^{a} \partial_{\beta} \chi^{a},
$$

where $g_{\alpha \beta}(X)$ is given by $(2.4)$.

In general, we can find a coordinate system where $g_{\alpha \beta}(X)$ is conformally flat. Then

$$
\sqrt{-g(X)} g^{\alpha \beta}(X)=\eta^{\alpha \beta}
$$

and (3.5) is independent of $X^{i}$. To perform such change of variables in the path integral one has to use a regularization covariant with respect to the induced metric $g_{\alpha \beta}(X)$. The integration over $\chi^{a}$ will produce quadratic and logarithmic divergences as well as a contribution to conformal anomaly (see, e.g., [13]). On the other hand, in critical string theory the divergences and the conformal anomaly are expected to cancel in the total expression for the partition function. Hence we may ignore a non-trivial 1-loop contribution from (3.5) as it should cancel when we add to it similar contributions from $A d S_{5}$ modes and the fermions. ${ }^{2}$ If we vary (3.5) with respect to $\partial \partial_{\tau} X^{0}$ to find the energy we obtain

$$
\begin{aligned}
& \frac{\partial L_{S^{5}}}{\partial \partial_{\tau} X^{0}}=-\frac{1}{4 \pi} \sqrt{-g(X)} \frac{\partial g^{\alpha \beta}(X)}{\partial \partial_{\tau} X^{0}} T_{\alpha \beta}(\chi), \\
& T_{\alpha \beta}(\chi)=\partial_{\alpha} \chi^{a} \partial_{\beta} \chi^{a}-\frac{1}{2} g_{\alpha \beta}(X) g^{\gamma \delta}(X) \partial_{\gamma} \chi^{a} \partial_{\delta} \chi^{a} .
\end{aligned}
$$

\footnotetext{
${ }^{2}$ Note that this cancellation is non-perturbative in $X^{i}$ as it is based on a regularization covariant with respect to the full metric $g_{\alpha \beta}(X)$ which cannot be imposed when we expand $g_{\alpha \beta}(X)$ in powers of $X$.
} 
Hence the above cancellation assumption can also be formulated as $\left\langle T_{\alpha \beta}\right\rangle=0$ for the full stressenergy tensor of all the bosonic and fermionic contributions. Note that on physical grounds one should not of course expect the $S^{5}$ fluctuations to contribute non-trivially to the energy since the string propagates only in $A d S_{5}{ }^{3}$

To finish this subsection let us find the propagator of the fields $\chi^{a}$ as we will need it in the next section. Using (3.6) we find that that $\chi^{a}$ have the action of massless fields in $A d S_{2}$

$$
S_{S^{5}}=\frac{1}{4 \pi} \int d \tau d \sigma\left[\left(\partial_{\tau} \chi^{a}\right)^{2}-\left(\partial_{\sigma} \chi^{a}\right)^{2}\right]
$$

Hence, the propagator $G_{0}\left(\tau_{1}, \sigma_{1} ; \tau_{2}, \sigma_{2}\right)$ satisfies

$$
\left(-\partial_{\tau_{1}}^{2}+\partial_{\sigma_{1}}^{2}\right) G_{0}\left(\tau_{1}, \sigma_{1} ; \tau_{2}, \sigma_{2}\right)=\delta\left(\tau_{1}-\tau_{2}\right) \delta\left(\sigma_{1}-\sigma_{2}\right) .
$$

Though this looks like the equation in flat space, $G_{0}$ has to satisfy the $A d S_{2}$ (half-plane) boundary conditions

$$
\left.G_{0}\right|_{\sigma_{1}=0}=0,\left.\quad G_{0}\right|_{\sigma_{2}=0}=0 .
$$

The corresponding solution is then given by

$$
G_{0}=-\frac{1}{2 \pi} \log \frac{\left(\sigma_{1}-\sigma_{2}\right)^{2}-\left(\tau_{1}-\tau_{2}\right)^{2}}{\left(\sigma_{1}+\sigma_{2}\right)^{2}-\left(\tau_{1}-\tau_{2}\right)^{2}} .
$$

Note that this is a function of a single variable $\ell$ which is (half) the geodesic distance in $A d S_{2}$

$$
\begin{aligned}
& G_{0}=-\frac{1}{2 \pi} \log \frac{\ell}{\ell+1}, \\
& \ell=\frac{1}{2} \frac{\left(\sigma_{1}-\sigma_{2}\right)^{2}-\left(\tau_{1}-\tau_{2}\right)^{2}}{2 \sigma_{1} \sigma_{2}} .
\end{aligned}
$$

\subsection{The contribution from $A d S_{5}$}

The $A d S_{5}$ part of the action is

$$
S_{A d S_{5}}=-\frac{\sqrt{\lambda}}{2 \pi} \int d \tau d \sigma \sqrt{-g(Y)},
$$

where $Y^{0}=X^{0}=\tau, Y^{4}=Z=\sigma$ and the transverse coordinates are given by (3.3). Then the induced metric will split as

$$
\begin{aligned}
& g(Y)=g(X)+g(X, y)+g(y), \\
& g_{\alpha \beta}(X)=g_{0 \alpha \beta}+\frac{1}{\sigma^{2}} \partial_{\alpha} X^{i} \partial_{\beta} X^{i}, \\
& g_{\alpha \beta}(X, y) \equiv h_{\alpha \beta}=\frac{1}{\lambda^{1 / 4}} \frac{1}{\sigma^{2}}\left[\partial_{\alpha} X^{i} \partial_{\beta} y^{i}+\partial_{\alpha} y^{i} \partial_{\beta} X^{i}\right], \\
& g_{\alpha \beta}(y)=\frac{1}{\sqrt{\lambda}} \frac{1}{\sigma^{2}} \partial_{\alpha} y^{i} \partial_{\beta} y^{i} .
\end{aligned}
$$

\footnotetext{
${ }^{3}$ This is obvious in conformal gauge but should be true also in static gauge assuming the conformal anomaly cancellation takes place.
} 
Here $g_{0 \alpha \beta}$ is the metric of $A d S_{2}$ and we used the static gauge conditions $X^{0}=\tau, Z=\sigma$ on the background fields and $y^{0}=0, y^{4}=0$ on the quantum fields.

Now we expand (3.15) to quadratic order in $y^{i}$ keeping the background $X^{i}$ arbitrary. We get

$$
\sqrt{-g(Y)}=\sqrt{-g(X)} \operatorname{det}^{1 / 2}\left[1+g^{-1}(X) \cdot g(y)+g^{-1}(X) \cdot h\right]
$$

where in the second factor we denote by $g(y)$ and $h$ the matrices $g_{\alpha \beta}(y)$ and $h_{\alpha \beta}$, by $g^{-1}(X)$ the matrix inverse to $g_{\alpha \beta}(X)$ and by $\cdot$ the matrix multiplication. We may then write (3.20) as

$$
\sqrt{-g(X)} \exp \left[\frac{1}{2} \operatorname{tr} \log \left(1+g(X)^{-1} \cdot g(y)+g^{-1}(X) \cdot h\right)\right]
$$

where the trace is over the worldsheet indices $(\alpha, \beta)$. Now we can expand (3.21) to quadratic order in $y$

$\sqrt{-g(Y)}=\sqrt{-g(X)}\left[1+\frac{1}{2} \operatorname{tr} g^{-1}(X) \cdot g(y)+\frac{1}{2} \operatorname{tr} g^{-1}(X) \cdot h-\frac{1}{4} \operatorname{tr}(g(X) \cdot h)^{2}+\frac{1}{8}\left(\operatorname{tr} g^{-1}(X) \cdot h\right)^{2}+\ldots\right]$.

This is the most general expression up to terms of order $y^{2}$ for an arbitrary background. The first term $\sqrt{-g(X)}$ is the classical action. The third term (linear in $h$ ) is linear in $y$ and hence it vanishes on the equations of motion for $X^{i}$. Let us consider the contribution coming from the second term

$$
S_{A d S_{5}}^{(1)}=-\frac{1}{4 \pi} \int d \tau d \sigma \frac{1}{\sigma^{2}} \sqrt{-g(X)} g^{\alpha \beta}(X) \partial_{\alpha} y^{i} \partial_{\beta} y^{i},
$$

where we used $g_{\alpha \beta}(y)$ in (3.17). This term is analogous to (3.5), so by our assumption discussed in the previous subsection its contribution should cancel against other similar contributions from the sphere and fermion terms. Hence, the non-trivial bosonic contribution should come from the last two terms in (3.22) quadratic in the metric $h_{\alpha \beta}$ in (3.18).

The fourth term in $(3.22)$ is

$$
S_{A d S_{5}}^{(2)}=\frac{\sqrt{\lambda}}{8 \pi} \int d \tau d \sigma \sqrt{-g(X)} g^{\alpha \beta}(X) g^{\gamma \delta}(X) h_{\beta \gamma} h_{\delta \alpha} .
$$

Since $h$ is linear in $X^{i}, h_{\beta \gamma} h_{\delta \alpha}$ is already quadratic in $X^{i}$ so we can replace $g(X)$ with the $A d S_{2}$ metric $g_{0}$.

Similarly, the last term in $(3.22)$ is

$$
S_{A d S_{5}}^{(3)}=-\frac{\sqrt{\lambda}}{16 \pi} \int d \tau d \sigma \sqrt{-g(X)} g^{\alpha \beta}(X) g^{\gamma \delta}(X) h_{\alpha \beta} h_{\gamma \delta} .
$$

Here we can also replace $g(X)$ with $g_{0}$. Both (3.24) and (3.25) contain only terms quadratic in $X^{i}$. Hence, the one-loop contribution comes from Feynman graph in Figure 1. 


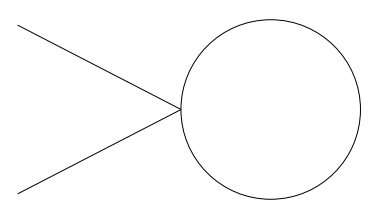

Figure 1: The Feynman graph describing the bosonic contribution to the one-loop effective action. The internal lines correspond to the quantum fields and the external lines are the background. Such a diagram is proportional to the Green's function at coinciding points.

The bosonic one-loop effective action is then given by

$$
e^{i \Gamma_{b}}=\int\left[d y^{i}\right] e^{i\left(S_{A d S_{5}}^{(0)}+S_{A d S_{5}}^{(2)}+S_{A d S_{5}}^{(3)}\right)}
$$

where $S_{A d S_{5}}^{(0)}$ is the free action given by (see eq. (3.23))

$$
S_{A d S_{5}}^{(0)}=\frac{1}{4 \pi} \int d \tau d \sigma \frac{1}{\sigma^{2}}\left[\left(\partial_{\tau} y^{i}\right)^{2}-\left(\partial_{\sigma} y^{i}\right)^{2}\right]
$$

We will need to find $\Gamma_{b}$ to order $X^{2}$. The propagator $\left\langle y^{i} y^{j}\right\rangle$ comes from the free action (3.27) and is thus diagonal. Let us denote

$$
\left\langle y^{i}\left(\tau_{1}, \sigma_{1}\right) y^{j}\left(\tau_{2}, \sigma_{2}\right)\right\rangle=2 \pi G_{2}\left(\tau_{1}, \sigma_{1} ; \tau_{2}, \sigma_{2}\right) \delta^{i j}
$$

To find $G_{2}$ we change variables $y^{i}=\sigma z^{i}$. Then

$$
S_{A d S_{5}}^{(0)}=\frac{1}{4 \pi} \int d \tau d \sigma\left[\left(\partial_{\tau} z^{i}\right)^{2}-\left(\partial_{\sigma} z^{i}\right)^{2}-\frac{2\left(z^{i}\right)^{2}}{\sigma^{2}}\right]
$$

This is the action of a massive field in $A d S_{2}$ with $m^{2}=2$. The equation for the propagator is then

$$
\left(-\partial_{\tau_{1}}^{2}+\partial_{\sigma_{1}}^{2}-\frac{2}{\sigma_{1}^{2}}\right) G_{2}=\delta\left(\tau_{1}-\tau_{2}\right) \delta\left(\sigma_{1}-\sigma_{2}\right)
$$

The solution to this equation satisfying the $A d S_{2}$ boundary conditions $\left.G_{2}\right|_{\sigma_{1}=0}=0,\left.G_{2}\right|_{\sigma_{2}=0}=0$ is given by

$$
G_{2}=\frac{1}{2 \pi}\left[-1-\frac{\sigma_{1}^{2}+\sigma_{2}^{2}-\left(\tau_{1}-\tau_{2}\right)^{2}}{4 \sigma_{1} \sigma_{2}} \log \frac{\left(\sigma_{1}-\sigma_{2}\right)^{2}-\left(\tau_{1}-\tau_{2}\right)^{2}}{\left(\sigma_{1}+\sigma_{2}\right)^{2}-\left(\tau_{1}-\tau_{2}\right)^{2}}\right]
$$

Note that it can be written as a function of the geodesic distance parameter $\ell$ defined in (3.14)

$$
G_{2}=\frac{1}{2 \pi}\left[-1-\left(\ell+\frac{1}{2}\right) \log \frac{\ell}{\ell+1}\right]
$$


Now let us consider $S_{A d S_{5}}^{(2)}$ and $S_{A d S_{5}}^{(3)}$. Using the explicit form of the matrix $h$ in (3.18) we obtain to quadratic order in $X^{i}$

$$
\begin{aligned}
S_{A d S_{5}}^{(2)} & =\frac{1}{8 \pi} \int d \tau d \sigma \frac{1}{\sigma^{2}} \eta^{\alpha \beta} \eta^{\gamma \delta}\left(\partial_{\beta} X^{i} \partial_{\gamma} y^{i}+\partial_{\beta} y^{i} \partial_{\gamma} X^{i}\right)\left(\partial_{\delta} X^{j} \partial_{\alpha} y^{j}+\partial_{\delta} y^{j} \partial_{\alpha} X^{j}\right) \\
& =\frac{1}{4 \pi} \int d \tau d \sigma \frac{1}{\sigma^{2}}\left[\left(\partial_{\alpha} X^{i} \partial^{\alpha} X^{j}\right)\left(\partial_{\beta} y^{i} \partial^{\beta} y^{j}\right)+\left(\partial_{\alpha} X^{i} \partial^{\alpha} y^{j}\right)\left(\partial_{\beta} X^{j} \partial^{\beta} y^{i}\right)\right] \\
S_{A d S_{5}}^{(2)} & =-\frac{1}{4 \pi} \int d \tau d \sigma \frac{1}{\sigma^{2}}\left(\partial_{\alpha} X^{i} \partial^{\alpha} y^{i}\right)\left(\partial_{\beta} X^{j} \partial^{\beta} y^{j}\right)
\end{aligned}
$$

where $\alpha, \beta$ are contracted using $\eta^{\alpha \beta}$. To compute $\Gamma_{b}$ to order $X^{2}$ we need to expand $\exp \left[i\left(S_{A d S}^{(2)}+\right.\right.$ $\left.\left.S_{A d S}^{(3)}\right)\right]$ to order $X^{2}$, i.e. we need to compute the expectation value of the operators in (3.33), (3.34). Since $\left\langle y^{i} y^{j}\right\rangle \sim \delta^{i j}$ we can simplify (3.33), (3.34) by taking only the diagonal contribution in $y^{i} y^{j}$. In other words, we can set $j=i$ and sum over $i$, i.e. keep only

$$
\begin{aligned}
\left(S_{A d S_{5}}^{(2)}+S_{A d S_{5}}^{(3)}\right)_{\text {trace }} & =\frac{1}{4 \pi} \int d \tau d \sigma \frac{1}{\sigma^{2}} \sum_{i}\left(\partial_{\alpha} X^{i} \partial^{\alpha} X^{i}\right)\left(\partial_{\beta} y^{i} \partial^{\beta} y^{i}\right) \\
& =\frac{1}{4 \pi} \int d \tau d \sigma \frac{1}{\sigma^{2}} \sum_{i}\left[\left(\partial_{\tau} X^{i}\right)^{2}-\left(\partial_{\sigma} X^{i}\right)^{2}\right]\left[\left(\partial_{\tau} y^{i}\right)^{2}-\left(\partial_{\sigma} y^{i}\right)^{2}\right]
\end{aligned}
$$

Replacing $y^{i}$ with $z^{i}=y^{i} / \sigma$ we get

$$
\left(S_{A d S_{5}}^{(2)}+S_{A d S_{5}}^{(3)}\right)_{\text {trace }}=\frac{1}{4 \pi} \int d \tau d \sigma \sum_{i}\left[\left(\partial_{\tau} X^{i}\right)^{2}-\left(\partial_{\sigma} X^{i}\right)^{2}\right]\left[\left(\partial_{\tau} z^{i}\right)^{2}-\left(\partial_{\sigma} z^{i}\right)^{2}-\frac{2}{\sigma} z^{i} \partial_{\sigma} z^{i}-\frac{\left(z^{i}\right)^{2}}{\sigma^{2}}\right]
$$

It is natural to define the $z$-dependent operator so that it does not contain first derivatives. For this we integrate the third term in the second bracket by part ignoring the terms with higher derivatives on $X$ as well as the boundary term, thus obtaining

$$
\begin{gathered}
\left(S_{A d S_{5}}^{(2)}+S_{A d S_{5}}^{(3)}\right)_{\text {trace }}=\frac{1}{4 \pi} \int d \tau d \sigma \sum_{i}\left[\left(\partial_{\tau} X^{i}\right)^{2}-\left(\partial_{\sigma} X^{i}\right)^{2}\right] \mathcal{O}^{i} \\
\mathcal{O}^{i}=\left(\partial_{\tau} z^{i}\right)^{2}-\left(\partial_{\sigma} z^{i}\right)^{2}-\frac{2\left(z^{i}\right)^{2}}{\sigma^{2}} .
\end{gathered}
$$

The one-loop effective action is then given by

$$
\begin{aligned}
& \Gamma_{b}=\frac{1}{4 \pi} \int d \tau d \sigma \sum_{i}\left[\left(\partial_{\tau} X^{i}\right)^{2}-\left(\partial_{\sigma} X^{i}\right)^{2}\right]\left\langle\mathcal{O}^{i}\right\rangle . \\
& \left\langle\mathcal{O}^{i}\right\rangle=\left.\left[\partial_{\tau_{1}} \partial_{\tau_{2}}-\partial_{\sigma_{1}} \partial_{\sigma_{2}}-\frac{2}{\sigma_{1} \sigma_{2}}\right] 2 \pi G_{2}\left(\tau_{1}, \sigma_{1} ; \tau_{2}, \sigma_{2}\right)\right|_{\tau_{2} \rightarrow \tau_{1} \rightarrow \tau, \sigma_{2} \rightarrow \sigma_{1} \rightarrow \sigma},
\end{aligned}
$$

where $G_{2}$ is given by (3.31). Substituting $G_{2}$ into (3.40) we find

$$
\left\langle\mathcal{O}^{i}\right\rangle=C_{1}+C_{2} \log \frac{\left(\sigma_{1}-\sigma_{2}\right)^{2}-\left(\tau_{1}-\tau_{2}\right)^{2}}{\left(\sigma_{1}+\sigma_{2}\right)^{2}-\left(\tau_{1}-\tau_{2}\right)^{2}}
$$


where

$$
\begin{aligned}
& C_{1}=\left.\frac{\left(\sigma_{1}+\sigma_{2}\right)^{2}\left(\sigma_{1}^{2}+\sigma_{2}^{2}\right)-2\left(2 \sigma_{1}^{2}+5 \sigma_{1} \sigma_{2}+2 \sigma_{2}^{2}\right)\left(\tau_{1}-\tau_{2}\right)^{2}+3\left(\tau_{1}-\tau_{2}\right)^{4}}{\sigma_{1} \sigma_{2}\left[\left(\sigma_{1}^{2}+\sigma_{2}^{2}\right)^{2}-\left(\tau_{1}-\tau_{2}\right)^{2}\right]^{2}}\right|_{\tau_{2} \rightarrow \tau_{1} \rightarrow \tau, \sigma_{2} \rightarrow \sigma_{1} \rightarrow \sigma}, \\
& C_{2}=\left.\frac{\left(\sigma_{1}-\sigma_{2}\right)^{2}-3\left(\tau_{1}-\tau_{2}\right)^{2}}{4 \sigma_{1}^{2} \sigma_{2}^{2}}\right|_{\tau_{2} \rightarrow \tau_{1} \rightarrow \tau, \sigma_{2} \rightarrow \sigma_{1} \rightarrow \sigma} .
\end{aligned}
$$

Taking the limit we get

$$
C_{1}=\frac{1}{2 \sigma^{2}}, \quad C_{2}=0
$$

so that (for any $i$ )

$$
\left\langle\mathcal{O}^{i}\right\rangle=\frac{1}{2 \sigma^{2}}
$$

and the bosonic one-loop effective action (3.39) is thus

$$
\Gamma_{b}=\frac{1}{8 \pi} \int d \tau d \sigma \frac{1}{\sigma^{2}}\left[\left(\partial_{\tau} X^{i}\right)^{2}-\left(\partial_{\sigma} X^{i}\right)^{2}\right] .
$$

Note that it has exactly the same form as the classical action (2.5) to order $X^{2}$. Also note that (3.46) is valid for an arbitrary classical background. In particular, on the Mikhailov's solution (2.8) it is given by

$$
\Gamma_{b}=\frac{1}{8 \pi} \int d \tau v^{i} a^{i}
$$

Now let us find the one-loop correction to the energy. We can do it by computing

$$
E_{b}=-\int d \sigma\left\langle\frac{\partial L_{b}}{\partial \partial_{\tau} X^{0}}\right\rangle
$$

For this we consider (3.24), (3.25) and restore the dependence on $X^{0}$ in the induced metric $g(X)$. That is, we replace in $(3.24),(3.25)$ the metric $g_{0}$ with the metric $\tilde{g}$ (see eq. (2.4))

$$
\tilde{g}_{\tau \tau}=-\frac{1}{\sigma^{2}}\left(\partial_{\tau} X^{0}\right)^{2}, \quad \tilde{g}_{\tau \sigma}=0, \quad \tilde{g}_{\sigma \sigma}=\frac{1}{\sigma^{2}}
$$

and set $\partial_{\tau} X^{0}=1$ after we take the derivative. Since all the dependence on $\partial_{\tau} X^{0}$ comes from the induced metric we get

$$
\begin{aligned}
& \frac{\partial S_{A d S_{5}}^{(2)}}{\partial \partial_{\tau} X^{0}}=\frac{\sqrt{\lambda}}{8 \pi} \int d \tau d \sigma \sqrt{-\tilde{g}} \frac{\partial \tilde{g}^{\mu \nu}}{\partial \partial_{\tau} X^{0}}\left[-\frac{1}{2} \tilde{g}_{\mu \nu} \tilde{g}^{\alpha \beta} \tilde{g}^{\gamma \delta} h_{\beta \gamma} h_{\delta \alpha}+2 \tilde{g}^{\alpha \beta} h_{\alpha \nu} h_{\beta \mu}\right], \\
& \frac{\partial S_{A d S_{5}}^{(3)}}{\partial \partial_{\tau} X^{0}}=-\frac{\sqrt{\lambda}}{16 \pi} \int d \tau d \sigma \sqrt{-\tilde{g}} \frac{\partial \tilde{g}^{\mu \nu}}{\partial \partial_{\tau} X^{0}}\left[-\frac{1}{2} \tilde{g}_{\mu \nu} \tilde{g}^{\alpha \beta} \tilde{g}^{\gamma \delta} h_{\alpha \beta} h_{\gamma \delta}+2 \tilde{g}^{\alpha \beta} h_{\alpha \beta} h_{\mu \nu}\right] .
\end{aligned}
$$

Using

$$
\frac{\partial \tilde{g}^{\tau \tau}}{\partial \partial_{\tau} X^{0}}=2 \sigma^{2}, \quad \frac{\partial \tilde{g}^{\tau \sigma}}{\partial \partial_{\tau} X^{0}}=0, \quad \frac{\partial \tilde{g}^{\sigma \sigma}}{\partial \partial_{\tau} X^{0}}=0
$$


and substituting the explicit expression for $h$ we obtain

$$
\frac{\partial S_{A d S_{5}}^{(2)}}{\partial \partial_{\tau} X^{0}}=\frac{1}{4 \pi} \int d \tau d \sigma \frac{1}{\sigma^{2}}\left[A+2 A_{\tau \tau}\right], \quad \frac{\partial S_{A d S_{5}}^{(3)}}{\partial \partial_{\tau} X^{0}}=-\frac{1}{4 \pi} \int d \tau d \sigma \frac{1}{\sigma^{2}}\left[B+2 B_{\tau \tau}\right]
$$

where (the worldsheet indices are contracted with $\eta^{\alpha \beta}$ )

$$
\begin{aligned}
& A=\left(\partial_{\alpha} X^{i} \partial^{\alpha} X^{j}\right)\left(\partial_{\beta} y^{i} \partial^{\beta} y^{j}\right)+\left(\partial_{\alpha} X^{i} \partial^{\alpha} y^{j}\right)\left(\partial_{\beta} X^{j} \partial^{\beta} y^{i}\right) \\
& B=\left(\partial_{\alpha} X^{i} \partial^{\alpha} y^{i}\right)^{2} \\
& A_{\alpha \beta}=\left(\partial_{\gamma} X^{i} \partial^{\gamma} X^{j}\right) \partial_{\alpha} y^{j} \partial_{\beta} y^{i}+\left(\partial_{\gamma} y^{i} \partial^{\gamma} y^{j}\right) \partial_{\alpha} X^{j} \partial_{\beta} X^{i} \\
& B_{\alpha \beta}=\left(\partial_{\gamma} X^{i} \partial^{\gamma} y^{i}\right)\left(\partial_{\alpha} X^{j} \partial_{\beta} y^{j}+\partial_{\alpha} y^{j} \partial_{\beta} X^{j}\right)
\end{aligned}
$$

Just like in the case of the effective action discussed above, in taking the vev in (3.53) only the diagonal terms in $y^{i} y^{i}$ will contribute. Thus in adding together the two expressions in (3.53) we can set $j=i$ and sum over $i$. We obtain

$$
\begin{gathered}
(A-B)_{\text {trace }}=\sum_{i}\left(\partial_{\alpha} X^{i} \partial^{\alpha} X^{i}\right)\left(\partial_{\beta} y^{i} \partial^{\beta} y^{i}\right)=\sum_{i}\left[\left(\partial_{\tau} X^{i}\right)^{2}-\left(\partial_{\sigma} X^{i}\right)^{2}\right]\left[\left(\partial_{\tau} y^{i}\right)^{2}-\left(\partial_{\sigma} y^{i}\right)^{2}\right] \\
\left(A_{\tau \tau}-B_{\tau \tau}\right)_{\text {trace }}=\sum_{i}\left(\partial_{\alpha} X^{i} \partial^{\alpha} X^{i}\right) \partial_{\tau} y^{i} \partial_{\tau} y^{i}+\sum_{i}\left(\partial_{\alpha} y^{i} \partial^{\alpha} y^{i}\right) \partial_{\tau} X^{i} \partial_{\tau} X^{i} \\
=-\sum_{i}\left[\left(\partial_{\tau} X^{i}\right)^{2}-\left(\partial_{\sigma} X^{i}\right)^{2}\right] \partial_{\tau} y^{i} \partial_{\tau} y^{i}-\sum_{i}\left[\left(\partial_{\tau} y^{i}\right)^{2}-\left(\partial_{\sigma} y^{i}\right)^{2}\right] \partial_{\tau} y^{i} \partial_{\tau} y^{i}
\end{gathered}
$$

Note that the sum of $(A-B)_{\text {trace }}$ and $\left(A_{\tau \tau}-B_{\tau \tau}\right)$ trace yields the Legendre transform of $(3.35)$ with respect to both background and quantum fields. Explicitly, summing the above two equations we obtain the two contributions to the energy. The first one is

$$
E_{b}=\frac{1}{4 \pi} \int d \tau d \sigma \frac{1}{\sigma^{2}} \sum_{i}\left[\left(\partial_{\tau} X^{i}\right)^{2}+\left(\partial_{\sigma} X^{i}\right)^{2}\right]\left\langle\left(\partial_{\tau} y^{i}\right)^{2}-\left(\partial_{\sigma} y^{i}\right)^{2}\right\rangle
$$

This term has exactly the same $y$-dependence as in (3.35). Changing the variables $y^{i}=\sigma z^{i}$ and integrating by parts we obtain

$$
E_{b}=\frac{1}{4 \pi} \int d \tau d \sigma \sum_{i}\left[\left(\partial_{\tau} X^{i}\right)^{2}+\left(\partial_{\sigma} X^{i}\right)^{2}\right]\left\langle\mathcal{O}^{i}\right\rangle
$$

where the operator $\mathcal{O}^{i}$ is given in (3.38). Using (3.45) we get

$$
E_{b}=\frac{1}{8 \pi} \int d \tau d \sigma \frac{1}{\sigma^{2}}\left[\left(\partial_{\tau} X^{i}\right)^{2}+\left(\partial_{\sigma} X^{i}\right)^{2}\right]
$$

However, eqs. (3.53)-(3.55) give rise to an additional unexpected correction of the form

$$
\tilde{E}_{b}=\frac{1}{4 \pi} \int d \tau d \sigma \frac{1}{\sigma^{2}} \sum_{i}\left[\left(\partial_{\tau} X^{i}\right)^{2}-\left(\partial_{\sigma} X^{i}\right)^{2}\right]\left\langle\partial_{\tau} y^{i} \partial_{\tau} y^{i}\right\rangle
$$


Let us carefully examine this term on the solution (2.8), (2.9), i.e. $X^{i}=x^{i}(\tau-\sigma)+\sigma \dot{x}^{i}(\tau-\sigma)$. First, on dimensional grounds, $\left\langle\partial_{\tau} y^{i} \partial_{\tau} y^{i}\right\rangle$ is independent of $\tau$ and $\sigma$. Then integrating by parts and using equations of motion we find

$$
\tilde{E}_{b} \sim \int \frac{d \sigma}{\sigma^{2}} \partial_{\tau}\left(X^{i} \partial_{\tau} X^{i}\right)-\int d \sigma \partial_{\sigma}\left(\frac{X^{i} \partial_{\sigma} X^{i}}{\sigma^{2}}\right)
$$

where the proportionality coefficient depends on $\left\langle\partial_{\tau} y^{i} \partial_{\tau} y^{i}\right\rangle$. Since the classical solution can be written as

$$
X^{i}=x^{i}-\sigma x^{i \prime}, \quad x^{i \prime}=\partial_{\sigma} x^{i}, \quad \partial_{\sigma} X^{i}=-\sigma x^{i \prime \prime}, \quad \partial_{\tau} X^{i}=-x^{i}+\sigma x^{i \prime \prime} .
$$

we get

$$
\begin{aligned}
\int d \sigma \partial_{\sigma}\left(\frac{X^{i} \partial_{\sigma} X^{i}}{\sigma^{2}}\right) & =\int d \sigma \partial_{\sigma}\left(x^{i \prime} x^{i \prime \prime}-\frac{1}{\sigma} x^{i} x^{i \prime \prime}\right) \\
\int \frac{d \sigma}{\sigma^{2}} \partial_{\tau}\left(X^{i} \partial_{\tau} X^{i}\right) & =\int \frac{d \sigma}{\sigma^{2}} \partial_{\sigma}\left(x^{i} x^{i \prime}\right)-\int \frac{d \sigma}{\sigma} \partial_{\sigma}\left[\left(x^{i \prime}\right)^{2}+x^{i} x^{i \prime \prime}\right]+\int d \sigma \partial_{\sigma}\left(x^{i \prime} x^{i \prime \prime}\right)
\end{aligned}
$$

where we used that $\partial_{\tau}$ acting on any function of $\tau-\sigma$ can be replaced with $-\partial_{\sigma}$. Now we can integrate by parts the second term in (3.63). The result will cancel the first term up to a boundary contribution. Hence, we get

$$
\int \frac{d \sigma}{\sigma^{2}} \partial_{\tau}\left(X^{i} \partial_{\tau} X^{i}\right)=-\int d \sigma \partial_{\sigma}\left[\frac{\left(x^{i \prime}\right)^{2}+x^{i} x^{i \prime \prime}}{\sigma}\right]+\int d \sigma \partial_{\sigma}\left(x^{i \prime} x^{i \prime \prime}\right)
$$

Substituting (3.62) and (3.64) in (3.60) we obtain

$$
\tilde{E}_{b} \sim \int d \sigma \partial_{\sigma}\left[\frac{\left(x^{i \prime}\right)^{2}}{\sigma}\right]=-\left.\frac{1}{\sigma}\left(x^{i \prime}\right)^{2}\right|_{\sigma=\epsilon \rightarrow 0}
$$

Expanding near $\sigma=\epsilon \rightarrow 0$ we have

$$
x^{i \prime}=-\dot{x}^{i}=-\dot{\mathrm{x}}^{i}-\epsilon \ddot{\mathrm{x}}^{i}+\cdots=-v^{i}-\epsilon a^{i}+\ldots,
$$

so that

$$
\tilde{E}_{b} \sim \frac{1}{\epsilon}\left(v^{i}\right)^{2}+2 v^{i} a^{i}
$$

These are precisely the first two terms in (2.21) which we have previously ignored in the classical expression. Hence, the whole contribution $\tilde{E}_{b}$ can be ignored and the bosonic one-loop correction is given by (3.58). 


\section{The one-loop contribution from the fermionic sector}

The quadratic fermionic action has the following form $[5,14]$

$$
S_{f}=\frac{1}{2 \pi} \int d \tau d \sigma\left[-2 i \sqrt{-G} G^{\alpha \beta} \bar{\theta} \rho_{\alpha} \nabla_{\beta} \theta+\epsilon^{\alpha \beta} \bar{\theta} \rho_{\alpha} \Gamma_{*} \rho_{\beta} \theta\right]
$$

where we imposed the $\kappa$-symmetry gauge $\theta^{1}=\theta^{2}=\theta$ (which is ghost-free). In (4.1) $\theta$ is a 32-component spinor satisfying the Majorana-Weyl condition, $G_{\alpha \beta}$ is the induced metric which to one-loop order is simply the background metric $g_{\alpha \beta}(X)$. The matrices $\rho_{\alpha}$ are

$$
\rho_{\alpha}=\Gamma_{A} E_{M}^{A} \partial_{\alpha} Y^{M}
$$

where $\Gamma_{A}, A=0, \ldots, 4$ are the $32 \times 32$ flat Dirac matrices satisfying

$$
\left\{\Gamma_{A}, \Gamma_{B}\right\}=2 \eta_{A B}, \quad \eta_{A B}=\operatorname{diag}(-1,1,1,1,1)
$$

$Y^{M}$ are the coordinates in $A d S_{5}$, which can be replaced by their classical parts: $Y^{0}=X^{0}=$ $\tau, Y^{4}=Z=\sigma, Y^{i}=X^{i} . E_{M}^{A}$ are the $A d S_{5}$ vielbeins

$$
E_{M}^{A}=\frac{1}{Z} \delta_{M}^{A}=\frac{1}{\sigma} \delta_{M}^{A}
$$

The matrices $\rho_{\alpha}$ are then given by

$$
\rho_{\tau}=\frac{1}{\sigma} \Gamma_{0}+\frac{1}{\sigma} \Gamma_{i} \partial_{\tau} X^{i}, \quad \rho_{\sigma}=\frac{1}{\sigma} \Gamma_{4}+\frac{1}{\sigma} \Gamma_{i} \partial_{\sigma} X^{i} .
$$

They satisfy

$$
\left\{\rho_{\alpha}, \rho_{\beta}\right\}=2 g_{\alpha \beta}(X) \text {. }
$$

For the string $A d S_{5}$ background the covariant derivative $\nabla_{\alpha}$ is given by

$$
\nabla_{\alpha}=\partial_{\alpha}+\frac{1}{4} \Omega_{M}^{A B} \Gamma_{A} \Gamma_{B} \partial_{\alpha} X^{M}
$$

i.e.

$$
\nabla_{\tau}=\partial_{\tau}-\frac{1}{2 \sigma} \Gamma_{0} \Gamma_{4}-\frac{1}{2 \sigma} \Gamma_{i} \Gamma_{4} \partial_{\tau} X^{i}, \quad \quad \nabla_{\sigma}=\partial_{\sigma}-\frac{1}{2 \sigma} \Gamma_{i} \Gamma_{4} \partial_{\sigma} X^{i}
$$

Finally, the matrix $\Gamma_{*}$ is given by

$$
\Gamma_{*}=i \Gamma_{0} \Gamma_{1} \Gamma_{2} \Gamma_{3} \Gamma_{4}, \quad \Gamma_{*}^{2}=1, \quad\left[\Gamma_{*}, \Gamma_{A}\right]=0
$$

We will use the basis where $\Gamma_{0}$ is antisymmetric and antihermitian while $\Gamma_{i}, \Gamma_{4}$ are symmetric and hermitian. Then $\Gamma_{*}$ is antisymmetric and hermitian. 
Figure 2: The Feynman graph describing the fermionic contribution to the one-loop effective action. The internal lines correspond to the fermionic loop and the external lines are the background.

Anticommuting Majorana spinors satisfy

$$
\begin{aligned}
& \bar{\theta}_{1} \Gamma_{A_{1} A_{2} \ldots A_{n}} \theta_{2}=\bar{\theta}_{2} \Gamma_{A_{1} A_{2} \ldots A_{n}} \theta_{1}, \quad n=3,7, \\
& \bar{\theta}_{1} \Gamma_{A_{1} A_{2} \ldots A_{n}} \theta_{2}=-\bar{\theta}_{2} \Gamma_{A_{1} A_{2} \ldots A_{n}} \theta_{1}, \quad n \neq 3,7 .
\end{aligned}
$$

In particular, it follows that $\bar{\theta} \Gamma_{A_{1} A_{2} \ldots A_{n}} \theta=0$ unless $n=3,7$. Note that the second term in (4.1) contains 7 gamma-matrices and, hence, is non-zero.

According to our discussion in the previous section, it is natural to assume that the kinetic term in (4.1) combines with (3.5) and (3.23) to cancel the conformal anomaly so that the entire non-trivial fermionic contribution should come from the second term in (4.1). Expanding this second term to order $X^{2}$ gives

$$
\frac{2}{\sigma^{2}} \bar{\theta}\left[i \Gamma_{0} \Gamma_{4} \Gamma_{*}-\Gamma_{4} \Gamma_{i} \Gamma_{*} \partial_{\tau} X^{i}+\Gamma_{0} \Gamma_{i} \Gamma_{*} \partial_{\sigma} X^{i}+\Gamma_{i j} \Gamma_{*} \partial_{\tau} X^{i} \partial_{\sigma} X^{j}\right] \theta
$$

Note that it contains linear terms in $X^{i}$ and, hence to find order $X^{2}$ terms we have to consider the Feynman graph in Figure 2. In flat space due to translational invariance in all directions we can go to the momentum space and single out the integral over the loop momentum. However, this cannot be done in $A d S_{2}$ since there is no translational invariance in the radial direction. Therefore, we will use a different approach.

Let is write the action (4.1) as

$$
S_{f}=-\frac{i}{\pi} \frac{1}{2 \pi} \int d \tau d \sigma \sqrt{-g(X)} \bar{\theta} \mathcal{D} \theta
$$

where $\mathcal{D}$ is the covariant (with respect to the induced metric $g_{\alpha \beta}(X)$ ) Dirac operator

$$
\mathcal{D}=g^{\alpha \beta} \rho_{\alpha} \nabla_{\beta}+\frac{i}{2 \sqrt{-g(X)}} \epsilon^{\alpha \beta} \rho_{\alpha} \Gamma_{*} \rho_{\beta}
$$

Formally "squaring" this operator leads to

$$
\hat{\mathcal{O}} \equiv \sqrt{-g(X)} \mathcal{D}^{2}=\sqrt{-g(X)} g^{\alpha \beta} \hat{\nabla}_{\alpha} \hat{\nabla}_{\beta}-\frac{1}{4} \sqrt{-g(X)} R^{(2)}-\sqrt{-g(X)}
$$


where $\hat{\nabla}$ contains extra connection term in addition to the $2 \mathrm{~d}$ spinor connection. ${ }^{4}$ Here $R^{(2)}$ is the curvature of the induced metric $g_{\alpha \beta}(X)$. The first term in (4.14) is a counterpart of (3.5) and (3.23). By our assumption the contribution from this term should cancel the similar bosonic contributions. The second term is a total derivative and its contribution can also be ignored. We shall thus assume that, in the first two terms, we can ignore the $X^{i}$-dependence so that we can effectively replace the metric $g(X)$ with the $A d S_{2}$ metric $g_{0}$. The non-trivial contribution then comes from the last term which is the square of the second term in (4.1). The operator $\hat{\mathcal{O}}$ we will write in the form

$$
\hat{\mathcal{O}}=\left(\eta^{\alpha \beta} \nabla_{0 \alpha} \nabla_{0 \beta}-\frac{1}{4} R_{0}-\frac{1}{\sigma^{2}}\right)-\left(\sqrt{-g(X)}-\frac{1}{\sigma^{2}}\right) \equiv \hat{\mathcal{O}}_{0}+\hat{\mathcal{O}}_{i n t} .
$$

Here $\hat{\mathcal{O}}_{0}$ is the free operator

$$
\hat{\mathcal{O}}_{0}=\eta^{\alpha \beta} \nabla_{0 \alpha} \nabla_{0 \beta}-\frac{1}{4} R_{0}-\frac{1}{\sigma^{2}}=-\partial_{\tau}^{2}+\partial_{\sigma}^{2}+\frac{1}{\sigma} \Gamma_{0} \Gamma_{4} \partial_{\tau}-\frac{3}{4 \sigma^{2}} .
$$

The operator $\hat{\mathcal{O}}_{\text {int }}$ contains interaction terms depending on the background

$$
\hat{\mathcal{O}}_{i n t}=\sqrt{-g(X)}-\frac{1}{\sigma^{2}}=-\frac{1}{2 \sigma^{2}}\left[\left(\partial_{\tau} X^{i}\right)^{2}-\left(\partial_{\sigma} X^{i}\right)^{2}\right]+\ldots
$$

Hence, to order $X^{2}$ the operator $\hat{\mathcal{O}}$ can be written as

$$
\hat{\mathcal{O}}=\hat{\mathcal{O}}_{0}+\frac{1}{2 \sigma^{2}}\left[\left(\partial_{\tau} X^{i}\right)^{2}-\left(\partial_{\sigma} X^{i}\right)^{2}\right]=\hat{\mathcal{O}}_{0}\left(1+\mathcal{G} \cdot \frac{1}{2 \sigma^{2}}\left[\left(\partial_{\tau} X^{i}\right)^{2}-\left(\partial_{\sigma} X^{i}\right)^{2}\right]\right)
$$

where $\mathcal{G}$ is the Green's function of the operator $\hat{\mathcal{O}}_{0}$. The fermionic contribution to the one-loop effective action is then given $b^{5}$

$$
\begin{aligned}
\Gamma_{f} & =\frac{1}{4} \operatorname{Tr} \log \hat{\mathcal{O}}=\frac{1}{4} \operatorname{Tr} \log \left(1+\mathcal{G} \cdot \frac{1}{2 \sigma^{2}}\left[\left(\partial_{\tau} X^{i}\right)^{2}-\left(\partial_{\sigma} X^{i}\right)^{2}\right]\right) \\
& =\frac{1}{8} \int d \tau d \sigma \frac{1}{\sigma^{2}}\left[\left(\partial_{\tau} X^{i}\right)^{2}-\left(\partial_{\sigma} X^{i}\right)^{2}\right] \operatorname{tr} \mathcal{G}(0)+\ldots
\end{aligned}
$$

where $\mathcal{G}(0)$ is the Green's function with coincident arguments and tr is the trace over the spinor indices.

Unfortunately, it appears to be complicated to find the Green's function of the operator (4.16) directly. Though the operator $\frac{1}{\sqrt{-g_{0}}} \hat{\mathcal{O}}_{0}$ is self-adjoint with respect to the measure

\footnotetext{
${ }^{4}$ In few simple cases of string backgrounds like parallel lines [5] or folded string [14] there is a non-trivial rotation of fermions that puts the derivative $\hat{\nabla}$ with induced connection into the form of $2 \mathrm{~d}$ spinor covariant derivative. That need not be the case if the background is general enough (an example is circular string with 3 spins in [15]). We thank S. Giombi for pointing out the relevance of this issue in the present case.

${ }^{5}$ We have the coefficient $1 / 4$ because we integrate over the real spinors $\theta$ and then square the Dirac operator.
} 
$\int d \tau d \sigma \sqrt{-g_{0}}$ its explicit form in (4.16) is not symmetric (or hermitian) in the usual sense. So we will follow an indirect approach. The equation for $\mathcal{G}\left(\tau_{1}, \sigma_{1} ; \tau_{2}, \sigma_{2}\right)$ can be written as follows

$$
\left[\rho_{0}^{\alpha} \nabla_{0 \alpha}+\frac{i}{\sqrt{-g_{0}}} \rho_{0 \tau} \rho_{0 \sigma} \Gamma_{*}\right]^{2} \mathcal{G}(1 ; 2)=\frac{1}{\sqrt{-g_{0}}} \delta\left(\tau_{1}-\tau_{2}\right) \delta\left(\sigma_{1}-\sigma_{2}\right) .
$$

Computing the square of the first-order operator in the left hand side of (4.20) and multiplying it by $\sqrt{-g_{0}}=\sigma^{-2}$ gives precisely (4.16). The result of (4.20) can be written in the form of a convolution

$$
\mathcal{G}(1 ; 2)=\int d \tau_{3} d \sigma_{3} \sqrt{-g_{0}\left(\tau_{3}, \sigma_{3}\right)} G_{f}(1 ; 3) G_{f}(3 ; 2),
$$

where $G_{f}$ is the fermionic Green's function satisfying the first order equation

$$
\left[\rho_{0}^{\alpha} \nabla_{0 \alpha}+\frac{i}{\sqrt{-g_{0}}} \rho_{0 \tau} \rho_{0 \sigma} \Gamma_{*}\right] G_{f}(1 ; 2)=\frac{1}{\sqrt{-g_{0}}} \delta\left(\tau_{1}-\tau_{2}\right) \delta\left(\sigma_{1}-\sigma_{2}\right) .
$$

Using the explicit expressions for $\rho_{0 \alpha}, \nabla_{0, \alpha}$ we can write (4.22) in the form

$$
\frac{1}{\sigma_{1}}\left[-\Gamma_{0} \partial_{\tau_{1}}+\Gamma_{4} \partial_{\sigma_{1}}+\frac{i}{\sigma_{1}} \Gamma_{0} \Gamma_{4} \Gamma_{*}\right] G_{f}(1 ; 2)=\delta\left(\tau_{1}-\tau_{2}\right) \delta\left(\sigma_{1}-\sigma_{2}\right) .
$$

Let us define $G_{f}=\sqrt{\sigma_{1} \sigma_{2}} G_{f}^{\prime}$. Then $G_{f}^{\prime}$ satisfies

$$
\left[-\Gamma_{0} \partial_{\tau_{1}}+\Gamma_{4} \partial_{\sigma_{1}}+\frac{i}{\sigma_{1}} \Gamma_{0} \Gamma_{4} \Gamma_{*}\right] G_{f}^{\prime}(1 ; 2)=\sqrt{\frac{\sigma_{1}}{\sigma_{2}}} \delta\left(\tau_{1}-\tau_{2}\right) \delta\left(\sigma_{1}-\sigma_{2}\right) .
$$

Due to the $\delta$-function we can replace $\sqrt{\frac{\sigma_{1}}{\sigma_{2}}}$ with 1 . Hence, we need to solve the equation

$$
D G_{f}^{\prime}(1 ; 2)=\delta\left(\tau_{1}-\tau_{2}\right) \delta\left(\sigma_{1}-\sigma_{2}\right), \quad D=-\Gamma_{0} \partial_{\tau}+\Gamma_{4} \partial_{\sigma}+\frac{i}{\sigma} \Gamma_{0} \Gamma_{4} \Gamma_{*},
$$

where $D$ acts on the first argument. Let us square this operator, i.e. consider the equation

$$
D^{2} G_{s}(1 ; 2)=\left[-\Gamma_{0} \partial_{\tau_{1}}+\Gamma_{4} \partial_{\sigma_{1}}+\frac{i}{\sigma_{1}} \Gamma_{0} \Gamma_{4} \Gamma_{*}\right]^{2} G_{s}(1 ; 2)=\delta\left(\tau_{1}-\tau_{2}\right) \delta\left(\sigma_{1}-\sigma_{2}\right) .
$$

If we know the solution to this equation $G_{s}$ then

$$
G_{f}^{\prime}(1 ; 2)=D G_{s}(1 ; 2), \quad G_{f}(1 ; 2)=\sqrt{\sigma_{1} \sigma_{2}} D G_{s}(1 ; 2) .
$$

Squaring $D$ gives explicitly

$$
\left[-\partial_{\tau_{1}}^{2}+\partial_{\sigma_{1}}^{2}-\frac{1}{\sigma_{1}^{2}}+\frac{1}{\sigma_{1}^{2}} i \Gamma_{0} \Gamma_{*}\right] G_{s}(1 ; 2)=\delta\left(\tau_{1}-\tau_{2}\right) \delta\left(\sigma_{1}-\sigma_{2}\right) .
$$

The matrix $i \Gamma_{0} \Gamma_{*}$ is symmetric and squares to 1 , so we can introduce the orthogonal projectors

$$
\mathcal{P}_{ \pm}=\frac{1}{2}\left(1 \pm i \Gamma_{0} \Gamma_{*}\right), \quad \mathcal{P}_{ \pm}^{2}=\mathcal{P}_{ \pm}, \quad \mathcal{P}_{+}+\mathcal{P}_{-}=1, \quad \mathcal{P}_{+} \mathcal{P}_{-}=\mathcal{P}_{-} \mathcal{P}_{+}=0
$$


Now let us look for a solution in the form

$$
G_{s}=\mathcal{P}_{+} G_{+}+\mathcal{P}_{-} G_{-} .
$$

It then follows that $G_{+}$and $G_{-}$should satisfy the following equations

$$
\begin{aligned}
& \left(-\partial_{\tau_{1}}^{2}+\partial_{\sigma_{1}}^{2}\right) G_{+}(1 ; 2)=\delta\left(\tau_{1}-\tau_{2}\right) \delta\left(\sigma_{1}-\sigma_{2}\right), \\
& \left(-\partial_{\tau_{1}}^{2}+\partial_{\sigma_{1}}^{2}-\frac{2}{\sigma_{1}^{2}}\right) G_{-}(1 ; 2)=\delta\left(\tau_{1}-\tau_{2}\right) \delta\left(\sigma_{1}-\sigma_{2}\right) .
\end{aligned}
$$

Thus $G_{+}$is the Green's function of a scalar field with $m^{2}=0$ which is given in (3.12). Similarly, $G_{-}$is the Green's function of a scalar field with $m^{2}=2$ which is given in (3.31). Hence, we finally obtain ${ }^{6}$

$$
G_{f}(1 ; 2)=\frac{1}{2 \pi} \sqrt{\sigma_{1} \sigma_{2}} D\left[\mathcal{P}_{+} G_{0}(1 ; 2)+\mathcal{P}_{-} G_{2}(1 ; 2)\right],
$$

where $D$ always acts on the first argument. Substituting $G_{f}$ into (4.21) gives

$$
\begin{aligned}
\mathcal{G}(1,2)=\frac{1}{4 \pi^{2}} \int d \tau_{3} d \sigma_{3} \sqrt{-g_{0}(3)} \sqrt{\sigma_{1} \sigma_{3}} \sqrt{\sigma_{3} \sigma_{2}} \\
\times \\
\times D\left[\mathcal{P}_{+} G_{0}(1 ; 3)+\mathcal{P}_{-} G_{2}(1 ; 3)\right] D\left[\mathcal{P}_{+} G_{0}(3 ; 2)+\mathcal{P}_{-} G_{2}(3 ; 2)\right] .
\end{aligned}
$$

We need to evaluate $\operatorname{tr} \mathcal{G}(1 ; 2)$ in the limit $\tau_{2} \rightarrow \tau_{1} \rightarrow \tau, \sigma_{2} \rightarrow \sigma_{1} \rightarrow \sigma$. The integral is of the same difficulty as the loop integral in Figure 2 and we were not able to evaluate it explicitly. We suggest the following indirect way to extract the finite contribution. First, we will write ${ }^{7}$

$$
D_{1}\left[\mathcal{P}_{+} G_{0}(1 ; 3)+\mathcal{P}_{-} G_{2}(1 ; 3)\right]=-D_{3}\left[\mathcal{P}_{+} G_{0}(1 ; 3)+\mathcal{P}_{-} G_{2}(1 ; 3)\right]
$$

where sub-indices on $D$ indicate the arguments on which it acts. Then we integrate by part to form $D_{3}^{2}$ ignoring all the additional terms. After integrating by parts we get

$$
D_{3}^{2}\left[\mathcal{P}_{+} G_{0}(3 ; 2)+\mathcal{P}_{-} G_{2}(3 ; 2)\right]=2 \pi \delta\left(\tau_{1}-\tau_{2}\right) \delta\left(\sigma_{1}-\sigma_{2}\right) .
$$

Then

$$
\operatorname{tr} \mathcal{G}(0)=\frac{1}{2 \pi} \operatorname{tr}\left(\mathcal{P}_{+} G_{0}+\mathcal{P}_{-} G_{2}\right)=\frac{1}{2 \pi} \cdot 16 \cdot \frac{1}{2}\left(G_{0}+G_{2}\right) .
$$

From the expressions for $G_{0}, G_{2}$ in $(3.13),(3.32)$ we find

$$
G_{0}+G_{2}=-1+(\ell+1) \log \frac{\ell}{\ell+1} .
$$

\footnotetext{
${ }^{6}$ Since $\theta$ is a Weyl spinor we should also introduce the Weyl projector. However, for simplicity, we will ignore it and use the fact that in the space of Weyl spinors the trace of the unit matrix gives 16 rather than 32 .

${ }^{7}$ Note that despite being a fermionic Green's function, $G_{f}$ is not explicitly antisymmetric. This is due to the boundary conditions in $A d S_{2}$, in particular, to the absence of the translational invariance along the $\sigma$-direction. Nevertheless, one can expect that in Feynman graphs one can use it as if it were antisymmetric.
} 
We see that there is a natural finite contribution -1 in $(4.37) .{ }^{8}$ Thus the finite part of $\operatorname{tr} \mathcal{G}(0)$ is given by

$$
\operatorname{tr} \mathcal{G}(0)=-\frac{4}{\pi}
$$

Substituting this into (4.19) gives

$$
\Gamma_{f}=-\frac{1}{2 \pi} \int d \tau d \sigma \frac{1}{\sigma^{2}}\left[\left(\partial_{\tau} X^{i}\right)^{2}-\left(\partial_{\sigma} X^{i}\right)^{2}\right] .
$$

This is the final expression for the one-loop correction to the order $X^{2}$ effective action coming from the fermionic sector.

To find the corresponding contribution to the energy we will start with the general expression for the one-loop effective action

$$
\Gamma_{f}=\frac{1}{4} \operatorname{Tr} \log \hat{\mathcal{O}}=\frac{1}{4} \operatorname{Tr} \log \left[1-\mathcal{G} \cdot\left(\sqrt{-g(X)}-\frac{1}{\sigma^{2}}\right)\right],
$$

and vary it with respect to $\partial_{\tau} X^{0}$. Taking the functional derivative gives

$$
-\frac{\partial \Gamma_{f}}{\partial \partial_{\tau} X^{0}}=\frac{1}{4} \operatorname{Tr}\left[\frac{1}{1-\mathcal{G} \cdot\left(\sqrt{-g(X)}-\frac{1}{\sigma^{2}}\right)} \cdot \mathcal{G} \cdot \frac{1}{2} \sqrt{-g(X)} g^{\tau \tau}(X) \frac{\partial g_{\tau \tau}(X)}{\partial \partial_{\tau} X^{0}}\right] .
$$

Expanding (4.41) to order $X^{2}$ produces two contributions. One comes from expanding $\sqrt{-g(X)}$ in the denominator. It is proportional to $\left(\partial_{\tau} X^{i}\right)^{2}-\left(\partial_{\sigma} X^{i}\right)^{2}$. This kind of term was discussed at the end of the previous section in eqs. (3.59)-(3.67) and was shown to be irrelevant. The relevant contribution comes from expanding the numerator $\sqrt{-g(X)} g^{\tau \tau}(X)$ which gives $-1-$ $\left.\frac{1}{2}\left[\partial_{\tau} X^{i}\right)^{2}+\left(\partial_{\sigma} X^{i}\right)^{2}\right]$. Using

$$
\frac{\partial g_{\tau \tau}(X)}{\partial \partial_{\tau} X^{0}}=-\frac{2}{\sigma^{2}}
$$

we find (ignoring the $X$-independent term)

$$
\left.-\frac{\partial \Gamma_{f}}{\partial \partial_{\tau} X^{0}}=\frac{1}{4} \operatorname{Tr}\left(\mathcal{G} \cdot \frac{1}{2 \sigma^{2}}\left[\partial_{\tau} X^{i}\right)^{2}+\left(\partial_{\sigma} X^{i}\right)^{2}\right]\right) .
$$

The one-loop fermionic contribution to the energy is then given by

$$
\left.\left.E_{f}=\frac{1}{8} \int d \sigma \frac{1}{\sigma^{2}}\left[\partial_{\tau} X^{i}\right)^{2}+\left(\partial_{\sigma} X^{i}\right)^{2}\right] \operatorname{tr} \mathcal{G}(0)=-\frac{1}{2 \pi} \int d \sigma \frac{1}{\sigma^{2}}\left[\partial_{\tau} X^{i}\right)^{2}+\left(\partial_{\sigma} X^{i}\right)^{2}\right],
$$

where we used eq. (4.38).

Let us now combine together the bosonic and fermionic one-loop contributions

$$
\begin{aligned}
& \left.\Gamma_{1-\text { loop }}=\Gamma_{b}+\Gamma_{f}=-\frac{3}{8 \pi} \int d \tau d \sigma \frac{1}{\sigma^{2}}\left[\partial_{\tau} X^{i}\right)^{2}-\left(\partial_{\sigma} X^{i}\right)^{2}\right], \\
& \left.E_{1-\text { loop }}=E_{b}+E_{f}=-\frac{3}{8 \pi} \int d \sigma \frac{1}{\sigma^{2}}\left[\partial_{\tau} X^{i}\right)^{2}+\left(\partial_{\sigma} X^{i}\right)^{2}\right] .
\end{aligned}
$$

\footnotetext{
${ }^{8}$ Note that one-half of the geodesic distance $\ell$ is a usual regulator in the point-splitting regularization in field theory in curved space-time, for a review see, e.g., [16].
} 
Evaluating these expressions on the Mikhailov's solution as in section 2 gives

$$
\begin{aligned}
& \Gamma_{1-\text { loop }}=-\frac{3}{8 \pi} \int d \tau v^{i} a^{i}, \\
& E_{1-\text { loop }}=-\frac{3}{4 \pi} \int d \tau\left(a^{i}\right)^{2}=2 \pi B_{1}(\lambda) \int d \tau\left(a^{i}\right)^{2},
\end{aligned}
$$

where the one-loop contribution to $B(\lambda)$ is given by (cf. (2.23))

$$
B_{1}(\lambda)=-\frac{3}{8 \pi^{2}} .
$$

This is the same as the second coefficient in (1.1). We thus provided a direct string perturbative check of the exact expression (1.1) proposed in [1].

\section{One-loop correction to the expectation value of a wavy Wilson line}

Let us now perform an analytic continuation along $\tau$ and $X^{0}$ and consider the Euclidean worldsheet and the Euclidean $A d S_{5}$ space. We will also work in the static gauge (2.2). A wavy Wilson line is a small deviation from a straight line $X^{0}=\tau, Z=\sigma$ in the transverse directions $X^{i}$ in Euclidean space $[8,9,10]$. To quadratic order in the transverse fields the Euclidean string action is

$$
S_{e}=\frac{\sqrt{\lambda}}{4 \pi} \int \frac{d \tau d \sigma}{\sigma^{2}}\left[\left(\partial_{\tau} X^{i}\right)^{2}+\left(\partial_{\sigma} X^{i}\right)^{2}\right],
$$

and the equations of motion are

$$
\partial_{\tau}^{2} X^{i}+\partial_{\sigma}^{2} X^{i}-\frac{2}{\sigma} \partial_{\sigma} X^{i}=0
$$

A wavy line is a solution to these equations with the boundary condition

$$
\left.X^{i}(\tau, \sigma)\right|_{\sigma=0}=\mathrm{x}^{i}(\tau)
$$

where $\mathrm{x}^{i}(\tau)$ is an arbitrary curve on the boundary. The precise form of the solution is given by $[10]$

$$
X^{i}(\tau, \sigma)=\int \frac{d \tau^{\prime}}{\pi} \mathrm{x}^{i}\left(\tau^{\prime}\right) \frac{2 \sigma^{3}}{\left(\left(\tau-\tau^{\prime}\right)^{2}+\sigma^{2}\right)^{2}} .
$$

Substituting it into the action gives ${ }^{9}$

$$
S_{e}=-\frac{\sqrt{\lambda}}{8 \pi^{2}} \int d \tau d \tau^{\prime} \frac{\left[\left(\dot{\mathrm{x}}(\tau)-\dot{\mathrm{x}}\left(\tau^{\prime}\right)\right]^{2}\right.}{\left(\tau-\tau^{\prime}\right)^{2}} .
$$

\footnotetext{
${ }^{9}$ It is straightforward to show that (5.5) is obtained starting with (5.1) (after dropping a total derivative). The minus sign in front of the Euclidean action in [10] is a misprint.
} 
The leading classical term in the expectation value of the wavy Wilson line is then given by

$$
\begin{aligned}
\left\langle W_{\text {wavy }}\right\rangle=e^{-S_{e}} & =1+\frac{\sqrt{\lambda}}{8 \pi^{2}} \int d \tau d \tau^{\prime} \frac{\left[\left(\dot{\mathrm{x}}(\tau)-\dot{\mathrm{x}}\left(\tau^{\prime}\right)\right]^{2}\right.}{\left(\tau-\tau^{\prime}\right)^{2}}+\ldots \\
& =1+\frac{1}{2} B_{0}(\lambda) \int d \tau d \tau^{\prime} \frac{\left[\left(\dot{\mathrm{x}}(\tau)-\dot{\mathrm{x}}\left(\tau^{\prime}\right)\right]^{2}\right.}{\left(\tau-\tau^{\prime}\right)^{2}}+\ldots,
\end{aligned}
$$

where we ignored the higher order terms in $\mathrm{x}^{i}$ and

$$
B_{0}(\lambda)=\frac{\sqrt{\lambda}}{4 \pi^{2}} .
$$

To the one-loop order the expectation value of $\left\langle W_{\text {wavy }}\right\rangle$ is

$$
\left\langle W_{\text {wavy }}\right\rangle=e^{-S_{e}-\Gamma_{e, 1-l o o p}},
$$

where $\Gamma_{e, 1-\text { loop }}$ is the Euclidean one-loop effective action computed on the solution (5.4). In the previous sections we computed the one-loop correction to the Minkowskian effective action (4.46) for an arbitrary classical background $X^{i}$. Its Euclidean analog can be found by a simple analytic continuation:

$$
\left.\Gamma_{e, 1-\text { loop }}=-\frac{3}{8 \pi} \int d \tau d \sigma \frac{1}{\sigma^{2}}\left[\partial_{\tau} X^{i}\right)^{2}+\left(\partial_{\sigma} X^{i}\right)^{2}\right] .
$$

Evaluating it on the solution (5.4) gives

$$
\Gamma_{e, 1-\text { loop }}=\frac{3}{16 \pi^{2}} \int d \tau d \tau^{\prime} \frac{\left[\left(\dot{\mathrm{x}}(\tau)-\dot{\mathrm{x}}\left(\tau^{\prime}\right)\right]^{2}\right.}{\left(\tau-\tau^{\prime}\right)^{2}} .
$$

Hence, to quadratic order in $\mathrm{x}^{i}$ we get

$$
\left\langle W_{\text {wavy }}\right\rangle=1+\frac{1}{2} B(\lambda) \int d \tau d \tau^{\prime} \frac{\left[\left(\dot{\mathrm{x}}(\tau)-\dot{\mathrm{x}}\left(\tau^{\prime}\right)\right]^{2}\right.}{\left(\tau-\tau^{\prime}\right)^{2}}+\ldots,
$$

where the one-loop correction to $B(\lambda)$ is given by

$$
B(\lambda)=B_{0}+B_{1}+\ldots, \quad B_{1}=-\frac{3}{8 \pi^{2}},
$$

i.e. is the same as in (4.49). Thus we checked that the one-loop correction to the expectation value of the wavy Wilson line is controlled by the same function $B(\lambda)$ as the energy radiated by a moving particle in agreement with the claim of [1].

\section{Acknowledgements}

We are grateful to M. Kruczenski for useful discussions. The work of E.I.B. was supported by the ARC Future Fellowship FT120100466. The work of A.A.T. was supported by the ERC Advanced grant No.290456 and also by the STFC grant ST/J000353/1. E.I.B. would also like to thank Theory Group at Imperial College and Center for Theoretical Physics at Tomsk State Pedagogical University where the part of the work was done for warm hospitality. 


\section{References}

[1] D. Correa, J. Henn, J. Maldacena and A. Sever, "An exact formula for the radiation of a moving quark in N=4 super Yang Mills," JHEP 1206 (2012) 048 [arXiv:1202.4455 [hep-th]].

[2] N. Drukker and V. Forini, "Generalized quark-antiquark potential at weak and strong coupling," JHEP 1106, 131 (2011) [arXiv:1105.5144 [hep-th]].

[3] A. Mikhailov, "Nonlinear waves in AdS / CFT correspondence," hep-th/0305196.

[4] M. Kruczenski and A. A. Tseytlin, "Wilson loops T-dual to Short Strings," arXiv:1212.4886 [hep-th].

[5] N. Drukker, D. J. Gross and A. A. Tseytlin, "Green-Schwarz string in $A d S(5) \times S^{5}$ : Semiclassical partition function," JHEP 0004 (2000) 021 [hep-th/0001204].

[6] M. Kruczenski and A. Tirziu, "Matching the circular Wilson loop with dual open string solution at 1-loop in strong coupling," JHEP 0805, 064 (2008) [arXiv:0803.0315 [hep-th]].

[7] C. Kristjansen and Y. Makeenko, "More about One-Loop Effective Action of Open Superstring in $A d S_{5} \times S^{5}, " J H E P$ 1209, 053 (2012) [arXiv:1206.5660 [hep-th]].

[8] A. M. Polyakov and V. S. Rychkov, "Gauge field strings duality and the loop equation," Nucl. Phys. B 581 (2000) 116 [hep-th/0002106].

[9] A. M. Polyakov and V. S. Rychkov, "Loop dynamics and AdS / CFT correspondence," Nucl. Phys. B 594 (2001) 272 [hep-th/0005173].

[10] G. W. Semenoff and D. Young, "Wavy Wilson line and AdS/CFT," Int. J. Mod. Phys. A 20 (2005) 2833 [hep-th/0405288].

[11] M. Chernicoff and A. Guijosa, "Acceleration and Energy Loss in N=4 SYM," AIP Conf. Proc. 1116 (2009) 285 [arXiv:0903.0306 [hep-th]].

[12] M. Chernicoff, J. A. Garcia and A. Guijosa, "A Tail of a Quark in N=4 SYM," JHEP 0909 (2009) 080 [arXiv:0906.1592 [hep-th]].

[13] N. D. Birrell and P. C. W. Davies, "Quantum Fields in Curved Space," Cambridge University Press, 1984.

[14] S. Frolov and A. A. Tseytlin, "Semiclassical quantization of rotating superstring in $A d S(5) \times S^{5}, "$ JHEP 0206 (2002) 007 [hep-th/0204226].

[15] S. Frolov and A. A. Tseytlin, "Quantizing three spin string solution in AdS(5) x S5," JHEP 0307, 016 (2003) [hep-th/0306130].

[16] D. V. Vassilevich, "Heat kernel expansion: User's manual," Phys. Rept. 388 (2003) 279 [hep-th/0306138]. 